\title{
Editorial
}

\section{On the Publication of the Special Issue}

Macromolecular Research has been launched in 1993 under the name of "Korea Polymer Journal" by the Polymer Society of Korea (PSK). The name of journal was changed to "Macromolecular Research" from 2002 after growing up to be included in Science Citation Index (SCI). Recently, the number of submitted papers and the impact factor of Macromolecular Research have been continuously raising up.

As a new activity to attract broad interests of the readers and to boost up the quality of the journal, the Editor-in-Chief Prof. Chulhee Kim decided to introduce the special issues in which thematic series of macromolecular research are introduced once in a while. The Editorial Board asked me to prepare a special issue on macromolecules for photonics and electronics, since I have been involved in the division of molecular electronics of PSK and active polymer research in an Engineering Research Center (ERC) of excellence supported by National Research Foundation of Korea.

During the first decade of the 21 st century, active polymers have emerged as the core materials for developing of innovative organic photonics and electronics based on micro patterning process. Recently, active polymer patterns have been prepared through various patterning process using molecular physics and chemistry. While the science of the synthesis and characterization for active polymers, such as electro, photo, or bioactive polymers, is interesting in both academic and application area, the control of the scale, shape, and dimension of their patterns provide new opportunity to develop organic devices and bionics with high performance and reliable functionality.

With this special issue, I invited leading scientists from Korea, Japan, China, Romania, Germany and USA who have contributed significantly to the macromolecular electronics and photonics. This issue includes feature articles and original research papers on processing and application potentials of active polymers and their patterning. Although I could not invite all the current hot research in this area, mainly due to the limited page in this volume, I believe this special issue on "Macromolecules for photonics and electronics" contains informative and creative research results, thus I hope that this cutting edge science will open a door to the discovery of macromolecules for photonics and electronics in the near future.

I express my deepest gratitude to the contributors for this issue and the Editors of Macromolecular Research for their editorial help and this opportunity.

Eunkyoung Kim

Director, Active Polymer Center for Pattern Integration (NRF)

Professor, Department of Chemical and Biomolecular Engineering, Yonsei University

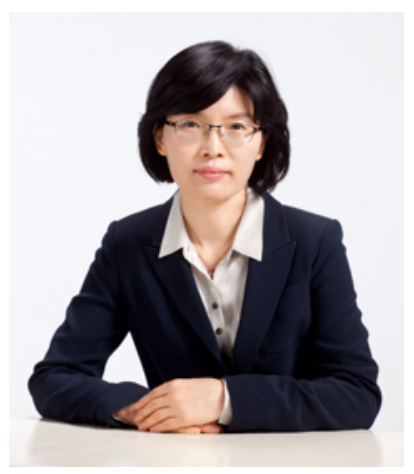

